\title{
El sociograma como instrumento que desvela la complejidad
}

\author{
Pedro Martin Gutierrez \\ Coordinador del Magister en Investigación, \\ Gestión y Desarrollo Local \\ (Universidad Complutense de Madrid)
}

A Mercedes.

A Josechu.

\begin{abstract}
«Para comenzar, uno ha de comenzar preguntándose qué es lo que comienza. Situación bastante más embarazosa que la del ciempiés que, antes de echar a andar, duda sobre qué pata moverá primero...»

Jesús Ibá̃̃ez.
\end{abstract}

Los instrumentos, las herramientas, los aparatos de que nos valemos cotidianamente, son versátiles y cuanto más simples, lo son en mayor medida. Un piolet (especie de pico con el que se auxilian, para progresar, aquellos alpinistas que marchan por nieve o hielo) ha servido y sirve, tanto para abrir la ruta a las cumbres montañosas, como para abrir nueces, como para abrir la cabeza a Trotsky. Pero, por sí mismo, este artefacto no es capaz de hacer ninguna de las operaciones antes mencionadas; su forma especializada es producto del ensayo en unas tareas y no en otras, pero en todo caso no conviene mitificar las bondades de un artefacto técnico.

De manera similar, el instrumento que me propongo mostrar operativamente puede ser aplicado con distintos fines, aunque por sí mismo, sin la habilidad y la

${ }^{1}$ El presente trabajo fue presentado como ponencia en el VI Congreso Español de Sociología (A Coruña, 24-26 de septiembre de 1998).

EMPIRIA. Revista de Metodología de Ciencias Sociales. N.² 2, 1999, pp. 129-151. 
intencionalidad del artesano, no nos va a mostrar -en este caso- aquello que de complejo tiene el medio social.

\section{LA MIRADA COMPLEJA}

Desde finales del pasado siglo, la concepción dominante en las ciencias, es puesta en cuestión desde distintos lugares. Hasta entonces la ciencia, entendida como el interés por describir relaciones causales entre hechos que acaecen, se apoyaba en leyes deterministas «...las cuales afirman que una serie dada de condiciones iniciales (situación antecedente) puede conducir a un y sólo un estado final (situación consecuente)» (Stent, 1990:73). Sin embargo, este determinismo se apoyaba en la marginación de todos aquellos sucesos singulares que, no siendo posible encuadrar en dichas leyes, pasaban a ser considerados como ruido, azar. El estudio más profundo de todo este ruido, en el campo de la física (la teoría cinética de los gases y la mecánica cuántica), llevó a enunciar otras leyes indeterministas «...[que] consideran que una serie dada de condiciones iniciales puede conducir a varios estados finales alternativos» (Stent,1990:73). El matemático Benoit Mandelbrot enuncia los postulados epistemológicos del indeterminismo, advirtiendo ya de un segundo estadio de indeterminismo que contemplase casos azarosos no encuadrados en el nuevo paradigma.

La observación por Illia Prigogine (Premio Nobel de Química, 1977) de procesos termodinámicos, en los que en estado de equilibrio las leyes deterministas actúan correctamente, pero que al alejarse de la situación de equilibrio, y una vez superado un estado caótico, adoptan nuevas estructuras ordenadas, espontáneas, con relación no lineal e impredecibles con exactitud (se puede predecir la aparición de una estructura disipativa, pero no los detalles de organización de esta estructura) le lleva a reconsiderar el dilema orden-desorden. Comprueba cómo en sistemas llamados complejos, alcanzado un cierto umbral en las fluctuaciones del sistema (hasta entonces amortiguadas por el propio sistema), un pequeño aporte de energía hace que el sistema adopte un nuevo equilibrio mientras se mantenga el aporte energético. Es lo que denomina estructuras disipativas. Se puede considerar que una estructura disipativa es «-Una fluctuación gigante mantenida por aportes de materia/energía - Una fluctuación amplificada a través de la interacción con el medio.»

Prigogine señala que las estructuras disipativas describen la aparición de modos de funcionamiento cualitativamente nuevos. Describirían una teoría de la creatividad. De esta forma la termodinámica y las ciencias humanas se ocuparían de lo mismo: estructuras creativas y activas... Las estructuras disipativas tienen dos características muy significativas: comunicación y adaptación.» (Manrique, 1990:75) ${ }^{2}$.

${ }^{2}$ Como mera curiosidad, dos ejemplos de estructuras disipativas. El fenómeno de las células de Bernard, por el que un líquido calentado progresivamente, va adquiriendo diversas formas de transmisión del calor en su seno, hasta que llega a un punto (rebasado el umbral de inestabilidad), en el que las moléculas se mueven formando células exagonales; es el orden a partir del caos. 
Volviendo a Mandelbrot, su descubrimiento de la fractalidad, en la que el modelo y el «objeto real» son idénticos, figuras fractales que no dejan puntos azarosos fuera de sí (porque han surgido de la misma manera, de forma iterativa), a diferencia del álgebra lineal, de las leyes universales y deterministas; en palabras de Jesús Ibáñez, la ley es siempre injusta porque no se ajusta a la realidad, sino que ajusta la realidad. Así, la concepción clásica subordina y violenta lo real (el objeto) a lo imaginario (la imagen) y esto a lo simbólico (el concepto). (Ibáñez, 1990:82-83). El incipiente paradigma complejo hace un nuevo planteamiento: no aporta soluciones a los problemas, sino que problematiza las soluciones, porque las distorsiones, las turbulencias que se manifiestan en los procesos hacen que las soluciones no sean simples, lineales, no están establecidas de manera determinista. Las soluciones abren nuevos campos problemáticos.

La relación entre orden (entendido como equilibrio) y desorden (identificado con el caos), es excluyente para la racionalidad de la ciencia clásica; el orden es lo que se ajusta a la operativización, lo que se deja aprehender por la visión lineal y determinista. Por el contrario el desorden, el caos, es sólo objeto de especulación, de aproximación estadística. La complejización de la mirada hacia el caos ha hecho surgir otras formas de concebir el orden, da sentido a la relación dialéctica orden/desorden.

El encumbramiento de la ciencia positiva precisa del concepto de objetividad; el sujeto debe ser apartado del camino científico porque perturba la observación. Así los términos ciencia y objetividad pasan a ser sinónimos y asimilados al de verdad («esto es científico, está científicamente comprobado», expresan, en lenguaje coloquial, la verdad irrefutable, se asimilan a la Palabra de Dios). Lo subjetivo, lo reflexivo, pertenece al campo de la filosofía, que no debe contaminar a la ciencia con su afición a especular; ciencia y filosofía son mutuamente excluyentes en sus modos de operar.

Frente al pensamiento clásico que excluye al sujeto de su actividad, el pensamiento complejo, consciente de que la realidad no es evidente y natural, sino construida socialmente, incorpora al sujeto como actor privilegiado. Se llega así a la certeza de que lo que se ha llamado ciencia, es también producto de un proceso social, que no es neutra. El campo de la ciencia pasa, desde la exclusión del sujeto, a su definición en función del sujeto: la actividad científica está compuesta por un sujeto y la realidad que éste trata de objetivar.

Pero el propio sujeto es parte de la realidad que de forma imposible y necesaria se empeña obsesivamente en desentrañar, en teorizar. Dice Jesús Ibáñez que «...desde que Velázquez pintó a Velázquez pintando el cuadro que pinta Velázquez, hasta que Patino filmo a Patino filmando el filme que filma Patino, abundan las obras de arte que son una pregunta por las condiciones de posibilidad de las obras de arte. El sujeto - 'absoluto', 'relativo'- se ha desvanecido: sólo queda la posibilidad de un sujeto 'reflexivo'. Son las tres posiciones en física: clásica, relativista y cuántica. El sujeto es interior a la representación, que es interior a lo

\footnotetext{
El segundo caso es el del reloj químico de Belusov-Zhabotinski. La mezcla de moléculas de distintos colores no provoca una reacción cuyo resultado sea un color intermedio, sino que a intervalos regulares la reacción da un color puro de un tipo y a continuación un color puro del otro tipo.
} 
representado». (Ibáñez, 1990:34). Las interpretaciones, las percepciones que tenemos del mundo están preñadas de valoraciones, de elementos que aportamos para llegar a tales interpretaciones o percepciones y de las cuales no podemos desposeernos, en un proceso circular, interno a sí mismo, sin salida desde dentro.

Las paradojas desconciertan al pensamiento lineal clásico. La prueba empírica y la teórica son paradójicas por autorreferentes: medir lo material con elementos materiales (Otero, 1971) (indeterminación de Heisenberg) y pensar el pensamiento (Barrow, 1997) (incompletitud de Gödel) nos hacen perseguir la quimera del conocimiento. «...la observación y la manipulación son reflexiones del objeto sobre sí mismo; el sujeto (observador/manipulador) es un espejo -refleja y refracta- que el universo se pone en el corazón» (Ibáñez, 1990: 35)

De la teoría de conjuntos han emanado tanto la teoría de grupos como la de redes: «... la teoría de las catástrofes es un traje en serie: primero se construye la teoría y luego se aplica. La teoría de objetos fractales es un traje a medida: se ha ido construyendo al hilo de sus aplicaciones. El mismo contraste se repite aquí: la teoría de grupos es un traje en serie; la teoría de redes, un traje a medida. Se ha ido construyendo un poco al buen tuntún...» (Ibáñez, 1990:19). El análisis de redes concibe el sistema social como redes de relaciones sociales, conjuntos de vínculos entre actores sociales (individuos, instituciones, grupos, clases...), no como conjuntos de entidades o individuos atomizados y desvinculados por el fraccionamiento estadístico, que es el enfoque del paradigma clásico dominante. La visión compleja en el análisis de redes se muestra al contemplar los vínculos entre actores sociales y no las meras relaciones diádicas, las normas o valores interiorizados o los atributos individuales que expresan las variables clásicas. Los conjuntos de vínculos, la posición en la estructura reticular o los vínculos entre vínculos (elemento clave en el análisis reticular), es una concepción de la vida social más compleja que las relaciones diádicas o los atributos personales.

Mientras el pensamiento simple -de primer orden- piensa el objeto (los sistemas observados), el pensamiento complejo -de segundo orden- piensa el pensamiento del objeto (los sistemas observadores) (Ibáñez, Jesús).

En nuestra condición de «seres que actúan», el principal centro de interés que orienta estas páginas es el de la transformación de la realidad, no el de la contemplación / explicación de las cosas. En este sentido, el pensamiento complejo induce a superar la «episteme» (en cuanto conocimiento científico/de la verdad), optando por la «fronesis» (el conocimiento de cómo funcionan las cosas) (Pearce, 1994:281); en vez de la teoría (del espectador), la praxis (del actor), la «fronesis» como sabiduría reflexiva que permite la transformación.

\section{EL PROCESO INVESTIGADOR COMO REDUCCIÓN PROGRESIVA DE DIMENSIONES Y PLANOS DE EXPRESIÓN}

Lo social es cambiante, impreciso, poliédrico... El proceso de definición/ aprehensión de la realidad es imposible y necesario (J. Ibáñez), por eso estamos 
empeñados en él. Si consideramos que la realidad social no es preexistente, sino construida socialmente, el proceso investigador no puede ser considerado como mero recopilador de datos, sino que es productor de información, creador de la realidad: «crear es bifurcar mediante dichos (inter-dic-ciones). Crear y elegir tienen la misma raíz» (J. Ibáñez , 1994:166) En la medida en que producimos esa información, es posible analizar nuestra ubicación (topológica) en el espacio social, nos definimos. Es desde esta posición desde la que «decimos», desvelamos, construimos, interpretamos,... los hechos sociales (M. Beltrán, 1991:170).

En la medida en que hacemos explícita nuestra ubicación topológica (siendo este paso consciente, reflexivo), estamos interviniendo en el proceso reflexivo de los actores/sujetos de la propia investigación.

En cuanto investigadores/productores de información, nuestro camino va concretando realidades multiformes, sirviéndonos para ello de herramientas guiadas y condicionadas por tácticas metodológicas y por estrategias epistemológicas y teóricas. En este camino hemos dejado jirones de realidad, renunciando a la contemplación de innumerables planos de conocimiento en aras de una mayor profundización en lo que es nuestro objetivo así construido.

La reducción de la perspectiva, desde la génesis del hecho social hasta la delimitación-ordenación-medición, es el tributo que ha de pagarse en el proceso investigador.

\section{PRIMER PASO (EPISTEMOLÓGICO). LOS NIVELES EN QUE SE CONSTRUYE E INVESTIGA LA REALIDAD SOCIAL: DE LA GÉNESIS AL ESPACIO EUCLÍDEO}

En términos generales, podemos establecer tres niveles de concreción de la realidad social (Ortí, 1994:93)(Conde, 1994:101), que corresponden a su vez con el continuo que va de lo más instituido/codificado a lo más instituyente/abierto:

* el de los «hechos», relativo a las construcciones sociales más cristalizadas;

* el de los «discursos», campo abierto a la reflexividad, a las relaciones con el lenguaje;

* por último el nivel de los «procesos motivacionales», el espacio más abierto, instituyente e inconcreto, que guarda el sentido, la intención de las acciones sociales.

Este progresivo movimiento de condensación y cristalización simbólica, de lo instituyente a lo instituido, podemos esquematizarlo en el siguiente cuadro (tomado de Conde 1994;103):

$\begin{array}{ll}\text { «A GÉNESIS SIMBÓLICA } & \rightarrow \begin{array}{l}\text { producción y apertura de un nuevo campo } \\ \text { simbólico y discursivo }\end{array} \\ \text { «B» CONSTITUCIÓN UNIVERSO } \rightarrow & \begin{array}{l}\text { desarrollo de un espacio simbolico multidi- } \\ \text { mensional y heterogéneo (Espacio de la poli - } \\ \text { hetero - topía) }\end{array}\end{array}$


«» CONSTITUCIÓN UNIVERSO $\rightarrow$ constitución de un espacio simbólico como DE LA SIGNIFICACIÓN semantización, articulado en torno a diferentes ejes de significación localmente homogéneos. Momento inicial de la medida (Espacios topológicos)

«D» CONSTITUCIÓN UNIVERSO $\rightarrow$ Desartollo de un espacio plano, homogéneo y DE LA MEDIDA unidimensional, en el que es posible desarrollar la medida cifrada (Espacio euclídeo).

Sobre este modelo (reductor) aquí presentado, vamos a mostrar los procesos epistemológicos comprendidos en cada nivel.

La primera etapa, la génesis, contemplaría dos pasos: uno la datación, la temporalización histórica, por la que situamos en el tiempo el origen del acontecimiento. El otro es la nominación (nombrar, dar nombre), acotamiento simbólico del hecho social así gestado ${ }^{3}$.

La segunda etapa es el espacio de la poli-hetero-topía (muchos planos multidimensionales -heterogéneos entre sí y dentro de sí- abordables sólo por su posición o localización). Este es el plano más abierto, instituyente, cualitativo, de la máxima dimensionalidad y en el que es posible establecer otros dos tipos de operaciones: la configuración y la valoración simbólicas. La primera contempla los primeros ejes de sentido, no mesurables pero que permitirán la ordenación a partir de elementos simbólicos (metáforas, estereotipos, prejuicios...).

La otra operación que permite esta etapa es la valoración simbólica. A partir de los ejes primarios que ha establecido la práctica anterior, podremos realizar un ordenamiento y por tanto establecer un rango entre los elementos. Estas operaciones continúan siendo exteriores al propio fenómeno social y por ende cargadas de valoraciones morales o ideológicas. Dependerá de estas diferentes interpretaciones o modos de construirlo el cómo lo vayamos a contemplar después.

Para nuestro propósito de crear mapas sociales de las relaciones entre actores, sociogramas, la siguiente etapa, que nos introduce en el espacio de las topologías ${ }^{4}$, es necesaria. Se trata de la configuración semántica. Esta operación tiene como virtudes que vamos a poder abstraer el fenómeno estudiado del contexto exterior, así como lograr una homogeneización interior que produzca las primeras condensaciones significantes, las cadenas de significantes que, no hay que olvidar, derivan de las operaciones anteriores, no son «naturales» o consustanciales al fenómeno en sí.

El camino de la concreción pasará posteriormente por las operaciones de jerarquizar elementos homogéneos y clasificarlos (estructuración significativa),

3 «... el coronel Aureliano Buendía habría de recordar aquella tarde remota en que su padre le llevó a conocer el hielo.[...] El mundo era tan reciente , que muchas cosas carecían de nombre, y para mencionarlas había que señalarlas con el dedo», García Márquez, Gabriel (1970): Cien años de soledad. Barcelona, Círculo de Lectores. (e.o. 1967) (p. 7).

4 TOPOLOGIA: «el estudio de los aspectos cualitativos de las formas espaciales o de las leyes de conexión, de la posición mutua y del orden de los puntos, rectas, superficies y cuerpos así como de sus puntos o agregados, hecha abstracción de sus relaciones de medida o magnitud. (Listing, citado en Conde, F., 1994:113) 
creando a partir de aquí el espacio euclídeo, en el que se ubican las técnicas cuantitativas de medición en ciencias sociales. Este espacio homogéneo que hemos ido desentrañando en el camino de la investigación, nos va a permitir dar cuenta de las dimensiones del fenómeno social expresadas por algoritmos matemáticos.

Hasta aquí, los diferentes pasos dados en el camino de la concreción métri$\mathrm{ca}$, nos han ido acotando espacios. El propósito que nos planteamos no es el de medir, en el sentido más elaborado y completo del término ${ }^{5}$, sino comprender las formas de las relaciones entre actores y ver las posibles reformulaciones de las mismas. Este propósito nos llevará a tomar el análisis de redes en sus procedimientos más elementales, coherentes con el plano de concreción métrica en el que nos hemos movido hasta ahora. En otras palabras, si nuestro nivel de medición llega a la delimitación de campos semánticos, sin hacer una clasificación de categorías homogéneas, no podremos «medir» en el sentido matemático, sino designar espacios y analizar sus características. El análisis apropiado ha de ser de tipo cualitativo.

\section{SEGUNDO PASO (METODOLÓGICO). EL ANÁLISIS DE REDES COMO APROXIMACIÓN AL CONOCIMIENTO DE LAS RELACIONES}

El análisis de redes (análisis reticular; network analysis) parte de la base de:

* se puede pensar la sociedad en términos de estructuras,

* que estas estructuras sociales se manifiestan en forma de relaciones entre actores sociales (sean estos actores, grupos, organizaciones, clases o individuos),

* que los conjuntos de vínculos o de relaciones sociales forman redes,

* que a su vez según sea la posición que los diferentes actores intervinientes ocupan en dichas redes, van a definir sus valores, creencias y comportamientos,

* el principio de análisis no son los individuos ni los grupos, sino las relaciones y las redes de relaciones, los grupos surgen de las redes de relaciones y están cruzados por la pertenencia de sus miembros a distintas redes (Garrido, 1996:141).

Las limitaciones más importantes del análisis reticular, es que es estático y descriptivo, pero de estos extremos hablaremos más adelante.

Del análisis de redes vamos a tomar para nuestros propósitos un elemento propio de la sociometría: el sociograma. "La técnica del sociograma consiste en representar gráficamente las relaciones interpersonales en un grupo de individuos mediante un conjunto de puntos (los individuos) conectados por una o varias líneas (las relaciones interindividuales). [...] (en el sociograma) ...las líneas empiezan

5 Para completar la argumentación sobre la «Reducción de la multidimensionalidad de lo real», desarrollada hasta aquí vid. Conde, 1994:98-119 y Ortí, 1994:85-95. 
a ser relaciones sociales de cualquier tipo y los puntos entidades sociales, que no se identifican necesariamente con los individuos» (Pizarro, 1990:147).

Las categorías que vamos a manejar en el análisis posterior van a ser tipos de relaciones, agrupación (conjuntos) de relaciones entre sujetos, densidades o discontinuidades en las relaciones y también diferencias en los contenidos de las relaciones entre los actores. Como se ve las categorías más clásicas en sociología, como los grupos de edad, género, territorio, etc. aquí no son considerados sino en función de las relaciones que mantienen, de las redes que forman con otros grupos o categorías sociales.

\section{TERCER PASO (TECNOLÓGICO). LA UTILIDAD DEL SOCIOGRAMA EN PROCESOS PARTICIPATIVOS}

Siguiendo el esquema de la investigación-acción participativa (I-A-P) que hace Villasante (1993:37), el sociograma tiene por misión representar gráficamente las relaciones de distinto tipo, que están presentes en un momento determinado, entre un conjunto de actores. Aquí el sociograma (lo instituyente) se enfrenta al organigrama (lo instituido, lo cristalizado) de manera que aporta a la investigación una perspectiva de lo que está pasando en el momento presente y por dónde deciden los implicados que han de desarrollarse las propuestas de actuación. Por el contrario, una de las limitaciones del sociograma, como hemos señalado más arriba, es su estatismo (en la acepción de estático) y su cualidad descriptiva, no explicativa.

Si nos remitimos al proceso de la I-A-P, en la segunda fase es en la que se negocia con los colectivos implicados, para construir el programa de actuaciones una vez realizado el diagnóstico. En este momento es preciso tener elaborado el mapa de las relaciones existentes, dado que de lo contrario no sabríamos con quién negociar eficazmente, a quién convocar y con qué alianzas y conflictos nos vamos a encontrar. En resumen, no sabemos qué terreno pisamos y por dónde pueden ir las propuestas de actuación, cuáles son los distintos intereses de los actores sociales y cómo se articulan sobre el terreno.

Por otra parte, el sociograma nos aporta, al igual que el diagnóstico previo, una evaluación «ex-ante» de la situación. Si hiciéramos una evaluación «expost» de la puesta en práctica de las propuestas, desarrolladas desde la investigación, y volviéramos a «mapear» ${ }^{6}$ el medio investigado, podríamos comprobar cómo se han articulado las redes sociales y (con mucha más dificultad) hasta qué punto ha influido el proceso de intervención en esta rearticulación.

El sociograma, se suele elaborar a partir de un test sociométrico y la correspondiente matriz de datos. Nosotros vamos a confeccionarlo mediante la triangulación de informaciones y el posterior análisis discursivo. Vamos a analizar la información que se recibe del proceso de reflexión de los sectores formales e

"A la realización del sociograma también le llamaremos «mapeo» 0 «mapear», tomando para ello la denominación que le da Paulo Freire. [Freire, P. (1993): «Interrogantes y propuestas», en Temas de Psicología Social, IV, 13, Buenos Aires. Citado por R. Villasante (1995b;189)]. 
informales de la población, de los dirigentes, técnicos, etc., y de los discursos sociales. Pero también va a ser una herramienta que vamos a desarrollar con los participantes en la investigación y que va a redundar en el proceso de reflexión y autoaprendizaje. Por último va a ser un elemento que contrastarán los actores y reformularán para, en la medida en que sea válido, emplearlo en la elaboración de los programas de actuación. Estos son los tres vértices con los que vamos a hacer los mapas de relaciones.

La contemplación de las redes ha de permitirnos, para ser eficaces, «de qué manera los de mayor confianza entre sí pueden ganar a los diferentes para enfrentar a los antagónicos»(Paulo Freire) y qué espacios son más propicios para desarrollar las propuestas de rearticulación de las redes.

\section{ELABORACIÓN Y VALIDACIÓN DEL SOCIOGRAMA POR LOS SUJETOS DE LA INVESTIGACIÓN}

En el análisis e interpretación de textos y discursos, el nivel autónomo (Ibáñez, 1986:323 y 333) alberga las diferentes posiciones desde las que se habla, las «hablas» de los diferentes hablantes. El análisis de los textos y los discursos nos va a permitir, por tanto, conocer las posiciones de diferentes individuos, grupos, actores, que se identifiquen con discursos o fracciones discursivas, sus relaciones y las características de éstas.

Una práctica participativa que se ha desarrollado (con buenos resultados) en este tipo de investigaciones ${ }^{7}$ es la de elaborar el «mapa de relaciones» con los propios sujetos colaboradores de la investigación, en una dinámica grupal. El método es sencillo. Consiste en explicarles a los colaboradores «las reglas del juego» (qué es un sociograma, para qué sirve y cómo se traza), desplegar un papel lo suficientemente amplio (papel continuo, de embalar, etc) y repartir rotuladores (si son de diferentes colores dan más oportunidades). El animador puede situar un punto de referencia, por ejemplo uno de los grupos o actores presentes en la reunión, y a partir de éste se van tejiendo las relaciones con otros grupos, instituciones o colectivos de la base social (jóvenes, mujeres, vecinos ...). Una vez puesta en marcha la dinámica, el animador no debe condicionar el desarrollo de la misma, dejando que los participantes modelen la técnica según sus gustos y necesidades.

Entre las «reglas del juego» ha de indicarse la conveniencia de reflexionar sobre tres elementos: la referencia acerca de quién o desde dónde se ejerce el poder, el nivel asociativo y la base social; estos niveles los representaremos por figuras geométricas o colores distintos. La composición puede realizarse en forma de pirámide, pero si surgen otras configuraciones, buenas son para el posterior análisis. Otra de las sugerencias es que las relaciones pueden ser de diferentes tipos: simples contactos (representadas por ejemplo por una línea continua), rela-

${ }^{7}$ Cf. VV.AA. (1995) Métodos de investigación social... Cuadernos de la Red, N. ${ }^{\circ} 4$. Red CIMS. Cf. diversos informes de las investigaciones desarrolladas en el Curso Postgrado «Magister en Investigación, Gestión y Desarrollo Local». Facultad de CC. P. y Sociología de la Universidad Complutense de Madrid. 
ciones estrechas y fluidas (trazo grueso o doble) y de conflicto (trazo con un aspa en medio). A partir de estos elementos suelen salir otros que simbolizan formas distintas de relacionarse. Por ejemplo, la relación con una institución puede ser fluida y crítica y los intervinientes deciden simbolizarla por un trazo doble, con un aspa en uno de ellos.

Esta práctica conlleva una contrastación inicial del sociograma, puesto que se elabora por acuerdos o por desacuerdos explicitados. Los manuales que explican estas técnicas suelen aplicar cuestionarios individuales, pero entendemos que la forma aquí expuesta es más fructífera en el tipo de investigación participativa que se realiza y con el análisis cualitativo empleado.

Tenemos por tanto dos fuentes iniciales de construcción del sociograma: el análisis de discursos y la colaboración de los sujetos de la investigación. Así elaborado, el mapa de relaciones será contrastado en las reuniones de «devolución» de la información y «negociación», es decir, cuando los resultados del diagnóstico son hechos públicos y cuando los actores interesados, los sujetos activos, se ponen a diseñar la programación de actividades que están dispuestos a desarrollar. Al tratarse de una investigación abierta, el sociograma puede ir enriqueciéndose en sucesivas etapas con nuevas aportaciones, que nos servirán para completar el análisis. Este sería el tercer elemento de triangulación del sociograma y un procedimiento de dinamización de la técnica.

No está de más poner de manifiesto, que en el diseño de las entrevistas iniciales, en la observación participante, en cualquiera de las técnicas cualitativas aplicadas, se deberá tener especial cuidado para no quedarse «enredado en una sola red». Es decir, que nuestros pre-supuestos iniciales o los primeros contactos con los actores sociales, no polaricen nuestra investigación en un solo espacio, dejando de lado los demás. La red así percibida será muy limitada y uniforme ${ }^{8}$.

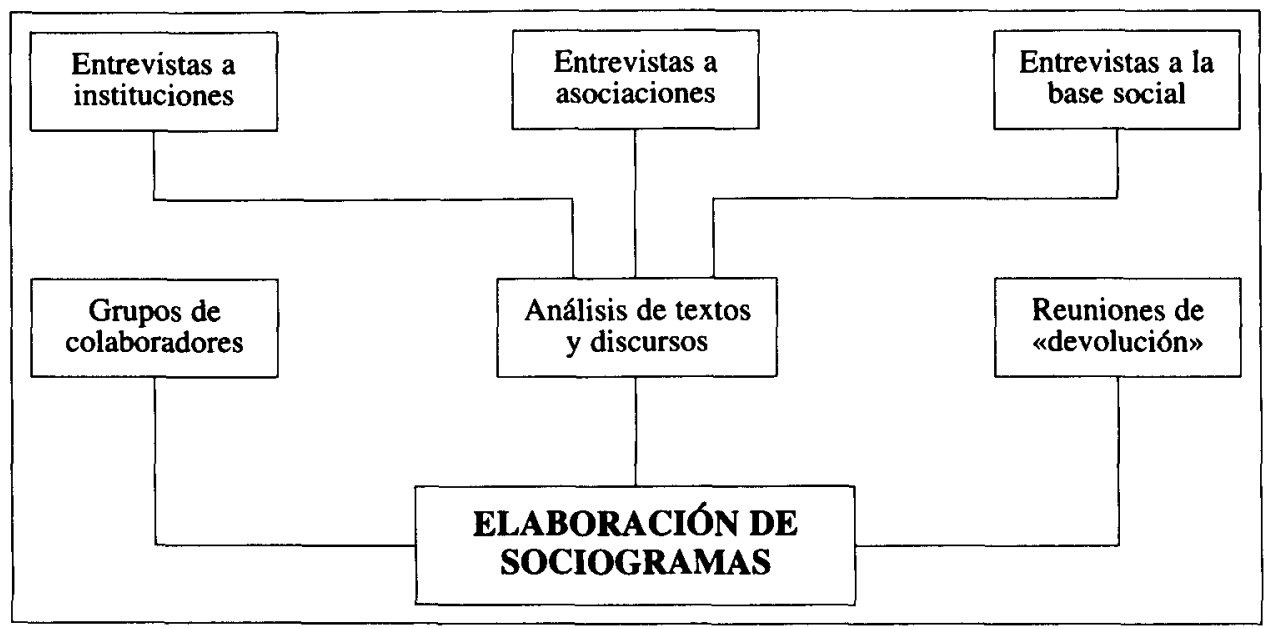

8 Vid. al respecto la crítica que realiza Mark Granovetter al trabajo de Herbert Gans sobre la comunidad italiana del lado Oeste de Boston. (Granovetter, 1973:1.373). 


\section{EL PROCESO DE ANÁLISIS Y LAS APLICACIONES DE LOS MAPAS SOCIALES}

El segundo punto de este trabajo plantea las sucesivas reducciones a que sometemos la multidimensionalidad de lo social en una investigación. Al realizar un sociograma con los actores sociales, lo que hacemos es seguir este mismo esquema expuesto.

En el momento inicial se opta por ubicar en tiempo y espacio los acontecimientos que vamos a reconstruir esquemáticamente, por tanto se van sentando las coordenadas de «aquello de lo que hablamos». A estos acontecimientos los nombramos en su nueva dimensión y los insertamos en un espacio simbólico, el de los actores del proceso. Por esto las relaciones aparecen cargadas de elementos simbólicos que posteriormente se irán perfilando todavía más: proximidad, afinidad, amiguismo, colaboración, buenas relaciones, encontronazos... También se delimitan los lugares de los grupos formales y los espacios de relación informal.

Configurar semánticamente el campo de intervención en el sociograma, implica que el fenómeno social que tratamos va a dar prioridad a la articulación interna frente a lo que le rodea. (Conde 1994) ${ }^{9}$.

Hay por tanto en esta etapa una abstracción del contexto, fijándose en las articulaciones interiores; vamos a definir los espacios semánticos diferenciados, homogéneos. En otras palabras, en el caso de un estudio, por ejemplo, sobre asociacionismo, vamos a fijarnos en los diferentes discursos y cómo se articulan en el mapa de relaciones, vamos a plasmar en términos de relaciones las diferentes formas de cooperación, afinidad, comunicación... de los grupos asociados, sus relaciones en sintonía con sus «hablas». Por eso, el contraste de los mapas que se van elaborando con los actores intervinientes, ubicará de nuevo las relaciones en un contexto pragmático, práxico.

Llegado el momento del análisis y dado el grado de concreción del espacio de medida, podemos hacer un análisis cualitativo, puesto que el grado de homogeneización no permite una cuantificación ${ }^{10}$.

Es conveniente tener en cuenta unos principios básicos:

* por lo general las relaciones son recíprocamente asimétricas, diferenciándose en contenido e intensidad,

* los miembros de la red se relacionan de manera directa e indirecta y es el conjunto del contexto estructural el que define una relación específica,

* las redes creadas por la estructura de relaciones no son arbitrarias,

* las relaciones pueden vincular a individuos así como a grupos y organizaciones.

9 Vid. F. Conde (1994) op. cit. p. 111. Es interesante la aplicación que hace el autor de cada uno de estos pasos, en el estudio de las dimensiones sociales del VIH/SIDA.

${ }_{10}$ De cara a los procesos participativos, en los que he puesto en práctica este instrumento, no es necesario desarrollar complejos análisis matemáticos, que por otra parte no nos aportarían una mejor aplicación del sociograma. Tampoco conviene complicar en exceso su elaboración e interpretación porque también se trata de transferir esta tecnología a los actores sociales, a los sujetos activos. 
El análisis lo podemos desarrollar considerando varios aspectos. En el análisis de redes se suelen aplicar dos perspectivas, bien complementadas o por separado. Desde una perspectiva "relacional», nos fijaríamos en las relaciones, tanto directas como indirectas, entre los actores; las cualidades de dichas relaciones, la intensidad, fortaleza, los conflictos, qué tipo de relaciones se mantienen (económicas, de intercambio, de solidaridad, clientelares, etc.).

Otro enfoque es el «posicional», en el que nos fijaremos en las formas que adquieren los diferentes conjuntos de actores. La mirada se centra ahora en las estructuras que se configuran.

Si en el primer enfoque podemos emplear la dimensión referencial de los textos y discursos (lo que dicen expresamente los actores, tanto de sí mismos como de otros o lo que dicen creer que dicen de ellos otros actores), con el fin de cualificar los diferentes tipos de relaciones, en este segundo enfoque emplearemos más la adscripción que hacen estos actores a los distintos discursos socialmente circulantes. Puede sernos útil emplear el modelo de los "conjuntos de acción» "11 y/o los bloques sociales, con el fin de caracterizar las posiciones homogéneas dentro del mapa.

Vamos a señalar a continuación algunos elementos a tener en cuenta para el análisis:

* la intensidad de las relaciones: debemos fijarnos en qué relaciones son dominantes en cada espacio, si las débiles o las intensas y esto en una doble dirección, tanto en horizontal como en vertical, entre pares y entre estratos ${ }^{12}$;

* la densidad de las relaciones: en un mapa suelen aparecer, como mínimo, un par de zonas densamente trabadas y diferenciadas. Estas zonas de alta densidad han de ser objeto de especial atención, tanto en sus características internas como en la relación que se mantiene entre ellas;

* observación de los elementos centrales: son los que mantienen relación con numerosos actores y el contacto entre éstos se mantiene a través del elemento central; sirve como aglutinador de un espacio densamente relacionado;

* observación de los elementos articuladores: es decir, aquellos que, sin ser necesariamente centrales, sin embargo ocupan una posición estratégica (por su actividad, prestigio, por la coyuntura...) como para unir varios conjuntos o rearticular la red. La centralidad tiene por tanto dos dimensiones: una es sinónimo del número de contactos que establece un actor, la otra está en función de la importancia de la posición del actor en aras de la articulación

11 Vid. R. Villasante, T. (Coord.) (1994b): «Clientelas y emancipaciones, una introducción metodológica», en Las ciudades hablan. Nueva Sociedad. Caracas. (pp. 25-47)

R. Villasante, T. (1991): Movimiento Ciudadano e Iniciativas Populares. HOAC. Madrid. (pp. 28-35)

12 Hemos de recordar la propuesta de configurar el mapa como una pirámide, con un vértice en el que se situaría el/los referentes del poder y una base social con todos los posibles grupos según variables poblacionales. El análisis vertical será del estrato intermedio (generalmente el asociativo o de grupos) con el poder y la base social. El horizontal será entre los distintos referentes de poder y entre los distintos grupos organizados. 
de la red. Este segundo aspecto considera la importancia de la mediación a través del actor central, sus cualidades como puente entre dos subgrupos que, de otra forma, estarían desconectados;

* los conflictos o ruturas de la red: fijándonos dónde se producen interferencias en la relación entre grupos o entre éstos y la base social y el carácter de estos conflictos;

* los espacios sin relacionar: es decir, aquellos lugares que hipotéticamente habrían de estar relacionados o en los que el establecimiento de relaciones facilitaría una posible actuación comunitaria, y en los que sin embargo no se da ningún contacto entre actores;

* las relaciones indirectas: que en un momento determinado puedan aclararnos un flujo de contactos entre actores no ligados directamente.

[ Sería una pregunta del tipo: si $\mathrm{A}->\mathrm{B}$ y $\mathrm{B}->\mathrm{C}=>$ ¿A->C ? ].

Este tipo de relaciones transitivas es considerado por autores expertos en redes como «la proposicion central en sociometría» ${ }^{13}$;

* observación de los puentes locales: esto tiene que ver con el análisis de las «relaciones débiles». A pesar de lo remoto de las relaciones entre dos actores, no por ello carecen de significado a la hora de ser analizadas. Si estas relaciones débiles unen dos sectores (que de otra manera estarían desconectados), los elementos que mantienen este tipo de vínculo reciben el nombre de puentes locales y son de gran importancia. Tomemos como ejemplo los procesos de difusión de información, en los que ha quedado demostrada la importancia de dichos puentes y su validez como articuladores de la red, tanto a nivel micro (de pequeños grupos, personales), como a nivel macro (de grupos sociales mayores, relación entre actores colectivos) ${ }^{14}$.

En diferentes trabajos se ha empleado un solo mapa ${ }^{15}$ de relaciones, con varias versiones o unidades parciales, sin embargo esto no superaba sus limitaciones mencionadas más arriba: su valor descriptivo y su estaticidad.

Si en vez de emplear un solo mapa empleamos varios, podremos captar distintos planos de la situación, comenzaremos a superponer fotograma a fotograma, hasta conseguir un incipiente movimiento, un proceso. La propuesta es hacer tres «cortes»:

13 Vid Granovetter, 1973:1.376, donde se refiere a trabajos de James Davis, Paul Holland y Samuel Leindhardt para determinar la estructura de la estratificación de un grupo. Para Granovetter, esta transitividad es probable si los vínculos son fuertes, escasa si son débiles e intermedia si uno es fuerte y el otro débil. La transitividad aparece por tanto como una función de la fuerza de los vínculos.

14 A este respecto es de suma importancia la línea de investigación sobre relaciones débiles que inicio Mark Granovetter y que, en los campos más variados, han continuado algunos otros.

Vid. Granovetter, M. (1973): «The strength of weak ties», en American Journal of Sociology; vol. 78, n. $^{\circ}$ 6. (pp. 1.360-1.380)

is Vid. Materiales del Curso Postgrado «Magister en Investigación, Gestión y Desarrollo Local», Facultad de CC. Políticas y Sociología de la Universidad Complutense de Madrid. En ellos se encontrarán diversas aplicaciones de esta técnica. 
* uno del pasado, por ejemplo, sobre un «analizador histórico»;

* un segundo en el momento presente, cómo ven los actores su realidad actual;

* el tercero en un escenario futuro, de cómo se quiere ver rearticulada la red por sus protagonistas y con qué actuaciones se lograría este propósito (hacia un «efecto mariposa»).

Si esto lo multiplicamos en tantos analizadores como podamos recrear y en tantos aspectos como nos permita la variada realidad social, tendremos un proceso en el que los propios actores representan su existencia.

De esta manera, el esquema previamente expuesto queda reformulado como sigue, añadiendo otras fases en las que los mapas de redes cumplen diferentes misiones, como la de comprobar y evaluar los efectos de recomposición de las redes, la de fijar objetivos de actuación, la de contrastar cómo las propuestas y programación de las mismas recomponen la estructura reticular...

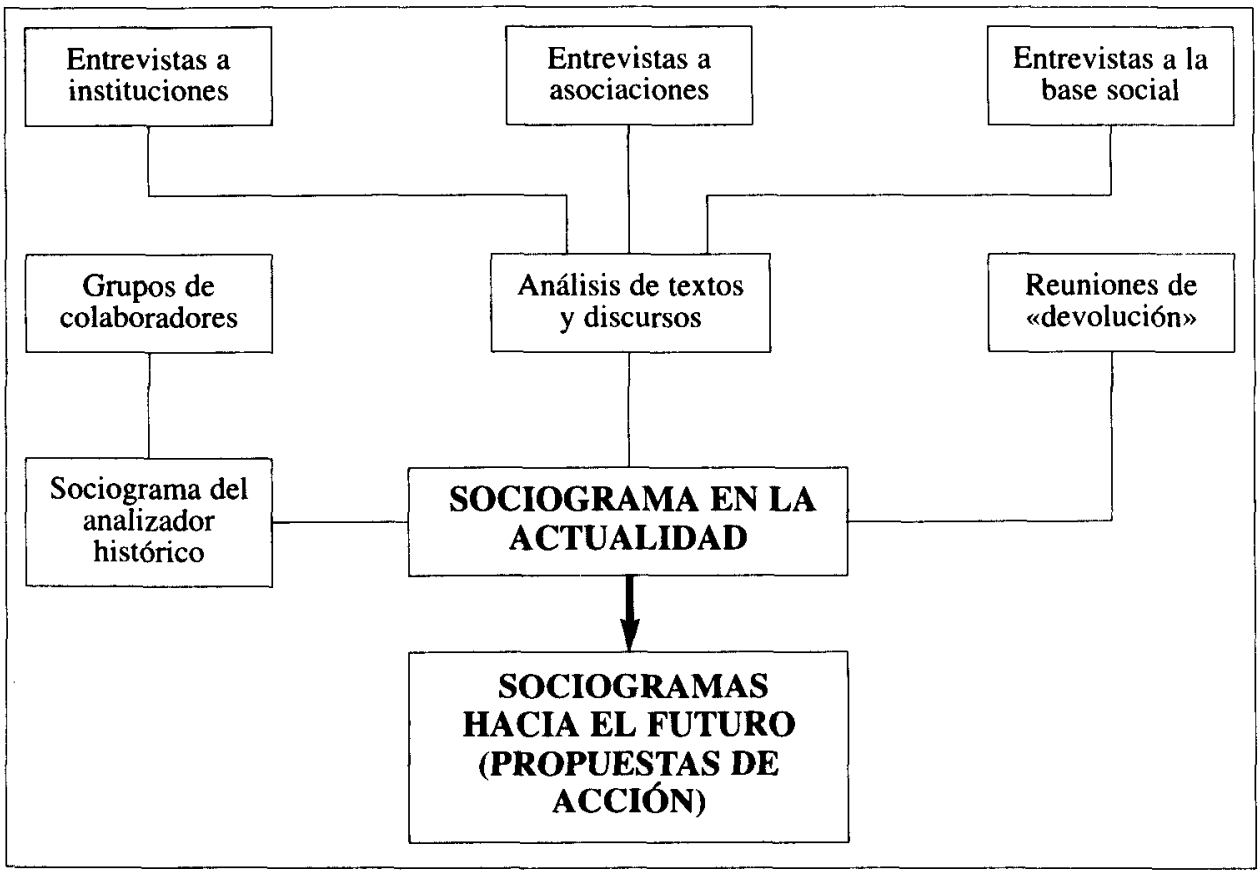

\section{ALGUNOS EJEMPLOS DE MAPAS SOCIALES}

A continuación se muestran un par de casos en los que se han empleado los sociogramas para la observación de la complejidad del espacio social, aunque con diferentes objetivos. 
En el primer caso (mapas 1 y 2) se trata de una investigación participativa en la que se contemplaba la situación de los hijos de inmigrantes (marroquíes en su mayoría), que estaban escolarizados en centros públicos del barrio de Lavapiés, en Madrid. Los problemas de esta población, los discursos acerca de su situación, tanto entre los inmigrantes como entre los nativos, hacen ver un panorama mucho más diverso y complejo del que en un principio y a través del discurso socialmente dominante aparece a diario. En el segundo caso ${ }^{16}$ (mapa 3 ) se aporta el mapa de relaciones entre actores (en este caso los actores son personas, no grupos ni instituciones, aunque algunos las representan) que ocupan posiciones singulares en la vida de un pequeño municipio guipuzcoano, Urnieta, con poco más de 5.000 habitantes.

En el MAPA 1 se muestran dos bloques discursivos centrales ${ }^{17}$ (los círculos más grandes), denominados «asimilacionista» $\mathrm{y}$ «multicultural» ${ }^{18}$. El entorno de estos bloques lo forman las diferentes fracciones discursivas de la población, quedando una a caballo entre ambos bloques (los niños españoles que muestran un discurso universal-nacionalista) y otra que no es encuadrable en ninguno de los dos bloques (la de los padres y niños inmigrantes aislados-cerrados, que demandan un respeto a su cultura y que no están dispuestos a ponerla en interacción con la del país de acogida ni con las de otros inmigrantes).

Las figuras rectangulares responden a los discursos de las instituciones u organizaciones presentes (centros educativos, asociaciones...), que se adscriben a los dos discursos centrales y a otras dos fracciones: una de carácter crítico con el discurso asimilacionista y otra denominada «acción desconcertante», que emana de los docentes que comienzan a plantearse la enseñanza desde postulados multiculturales y perciben las contradicciones propias de este nuevo planteamiento. Estas dos fracciones (relacionadas porque surgen de la fractura del mismo discurso) forman un «puente local» (en términos de Granovetter) que constituye el enlace por donde fluye la comunicación entre los dos bloques discursivos. Véase por tanto la importancia estratégica de ambas fracciones.

Los triángulos representan la percepción que los actores tienen de quién encarna el poder, de quién conforma los discursos dominantes. En este caso son departamentos de la administración central del Estado y los medios de comunicación social.

El MAPA 2 representa cómo se vería la red social estudiada, si la propuesta, que plantean una parte de los actores sociales, de hacer una programación de actividades que contemple y refuerce los valores de multiculturalidad, tanto en los centros educativos como en el barrio, se pusiera en práctica. Este mapa representa el objetivo de la acción propuesta, la imagen de futuro: aglutinar las fracciones discursivas más proclives a la multiculturalidad en el barrio de Lavapiés, los actores presentes y con la participación de los centros educativos, en los que (también) se socializan los hijos de los inmigrantes.

16 Vid. Bautista, Antoni y Roviera, Bru (1998): «Nacionalistas vascos. La familia de los silencios», Magazine-La Vanguardia, 8 de febrero, (pp. 38-47).

17 Vid. Martín Gutiérrez et alii (1995a:31-38).

$18 \mathrm{El}$ abanico discursivo, el marco conceptual empleado se debe originalmente al Colectivo loé, de quienes lo tomamos para la realización de este trabajo (Colectivo IOÉ 1987:320-323). 


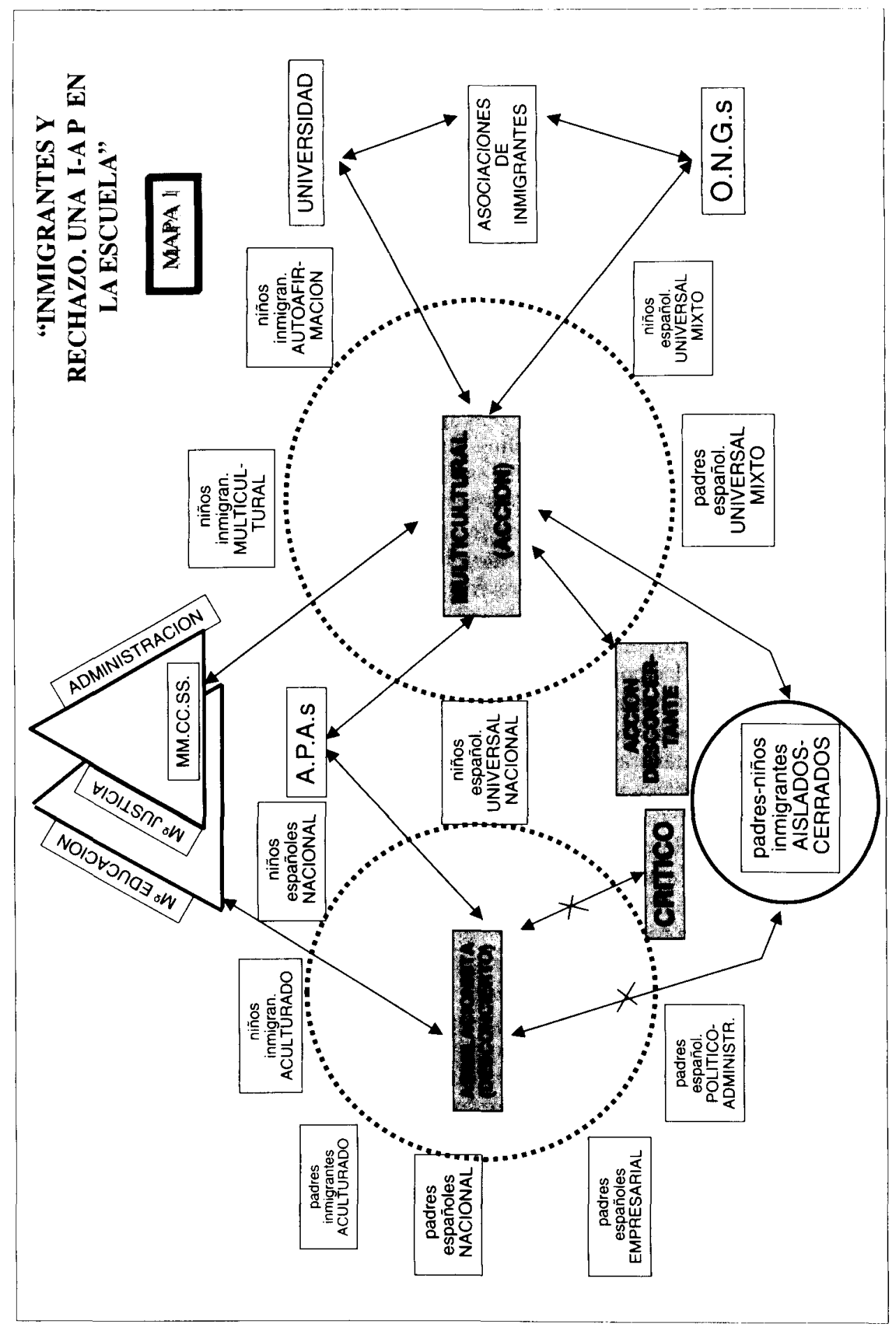




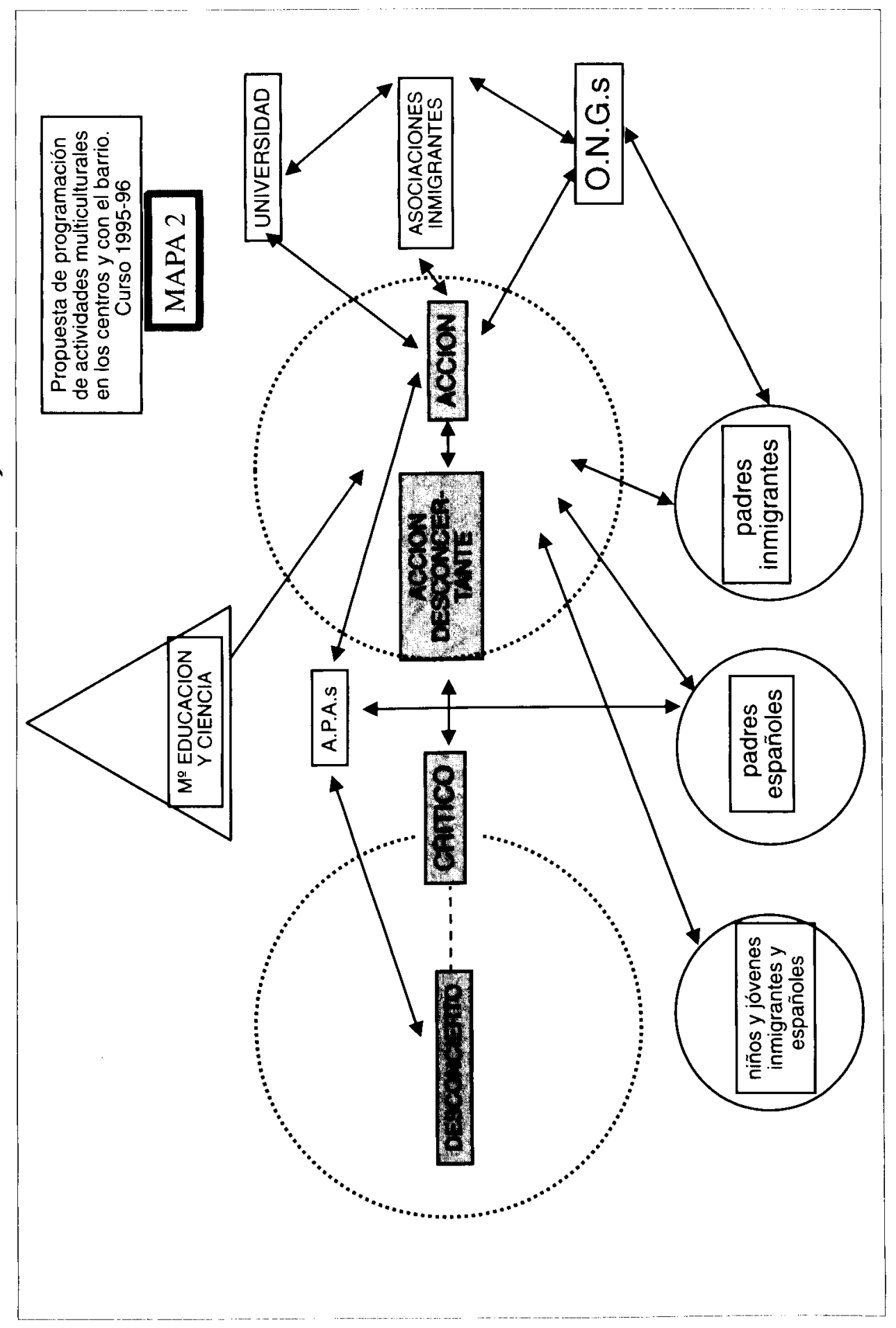




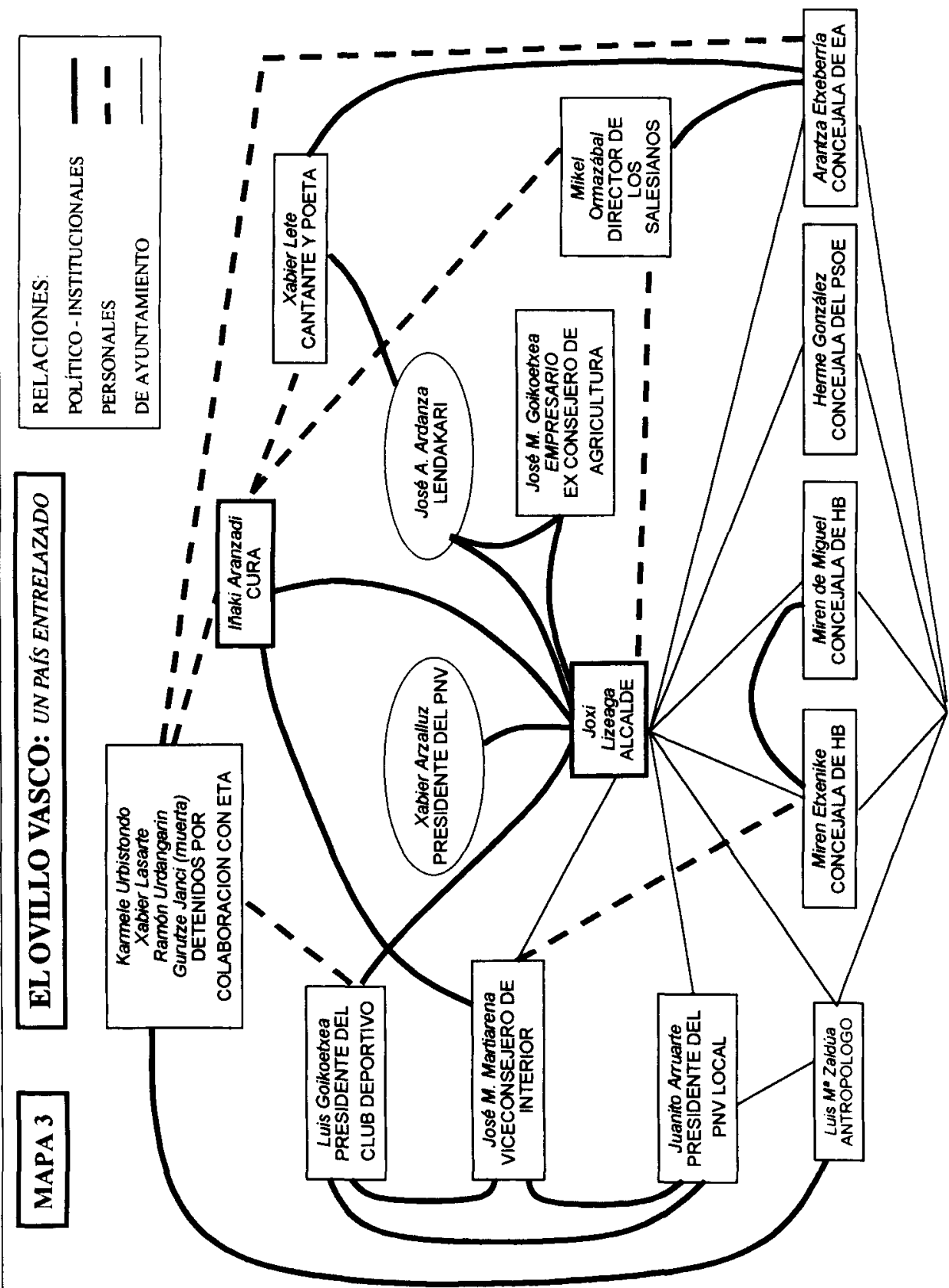


El MAPA 3 muestra la trama de relaciones observadas con el objeto de «...comprender lo que sucede en Euskadi» (Bautista y Roviera, 1998:46). El artículo periodístico narra las múltiples formas de relación existentes entre estos actores, desmontando línea a línea el discurso dominante acerca del «problema vasco», a la par que señala, en un análisis tal vez demasiado breve, los actores centrales y las distintas centralidades en la vida del municipio ${ }^{19}$ (vid. el subrayado en la nota a pie de página).

Hay que llamar la atención sobre dos aspectos de este mapa: en primer lugar la posibilidad de trasladar a nivel macro (de todo el espacio social vasco: «...comprender lo que sucede en Euskadi», como dicen los autores) los rasgos estructurales de esta relación de actores. En segundo término el papel cualitativamente central (como «puente local»), que se asigna al cura como mediador entre los espacios cuantitativamente más relacionados: el Ayuntamiento y el grupo de detenidos. También hay que tener en cuenta el variado tipo de relaciones que se muestran, a pesar de que en el mapa se señalen sólo tres: político-institucionales, de Ayuntamiento y personales ${ }^{20}$. Una de las potencialidades de estos mapas es mostrar la complejidad de la vida social, que suele verse encorsetada en discursos simples y simplistas, como representa el discurso socialmente dominante; en ambos casos dicotómico, lineal, sin aristas. En el primer caso entre «inmigrantes» y «nativos»; en el segundo, el «problema vasco» se debate entre «demócratas» y «violentos» o entre «vascos» $y$ «no vascos».

19 «EL PAÍS ENTRELAZADO. El padre de la concejal de HB Miren Etxenique fue íntimo amigo del actual viceconsejero de Interior con mando directo en la Ertzaina José A. Martiarena. Miren fue, a su vez, alumna del alcalde, Joxi Lizeaga , cuando éste ejercía de maestro, y Joxi fue discípulo político de Martiarena, que fue su antecesor en la alcaldía. Karmele Urbistondo, que acaba de salir de la cárcel tras cumplir condena por colaboración con ETA, también fue alumna de Joxi y fue secretaria del antropólogo Luis M. ${ }^{\mathrm{a}}$ Zaldúa, técnico de Cultura del Ayuntamiento. Xabier Lasarte, otro de los condenados, tuvo una fluida relación personal con el que fue director del club deportivo, el militante del PNV Luis Goikoetxea, y estudió en los Salesianos. Arantza Etxeberría, concejala de EA, fue secretaria del cantautor Xabier Lete, cuando éste era diputado de Cultura y el presidente de la Diputación era el actual lendakari, Ardanza. Arantza, a su vez, fue muy amiga de la novia de uno de los detenidos. El Ayuntamiento es el principal foco cuantitativo de relaciones, pero el cura Iñaki Aranzadi, es el cualitativamente más relacionado: es el único que tiene relación con todos los otros sujetos/actores más relacionados del pueblo, el alcalde, el grupo de detenidos y el cantautor Xabier Lete. El menos relacionado es el concejal del PSOE y el PP ni existe. Sin esta red personal es imposible conocer lo que sucede en Euskadi. Proyectándolo hacia arriba, salen tres nombres importantes de la política vasca: el viceconsejero Martiarena, que es del pueblo; el presidente del PNV y el lendakari. El PNV quedaría como un gran común denominador imprescindible para poder hilar desde el ovillo vasco» (Bautista y Roviera, 1988:46) (La cursiva es mía).

${ }^{20}$ El contenido de las relaciones contempladas varían dependiendo de los autores y las necesidades de la investigación. Por ejemplo F. Rodríguez, en su Tesis Doctoral, define las siguientes clases: ayudas y subvenciones, divulgación, concertación y conflicto (Rodríguez, 1997:456). Otro autor, F. Requena, emplea las afectivas, normativas y de intercambio (Requena, 1996:24). En resumen, se encuadran en los tres subsistemas de intercambio que señala Lévi-Strauss: libidinal, material y lingüistico. 


\section{INTENTANDO SUPERAR LIMITACIONES: RELOJES Y RUIDO}

Los trabajos empíricos en los que he sometido a prueba el sociograma, han estado relacionados principalmente con asociacionismo y se ha manejado dentro de metodología participativas. Teniendo en cuenta este contexto quiero hacer algunas reflexiones finales.

Los sociogramas, por lo general, se elaboran a partir de test sociométricos, que no contemplan (salvo si hay intencionalidad expresa) los vínculos débiles entre actores. Quiero hacen hincapié en la importancia de este tipo de relaciones. En primer lugar se debe resaltar su utilidad como canal de divulgación de información y opinión, pero también como factor de cambio y de cohesión social.

También quiero hacer notar la importancia de analizar los puentes locales, que con frecuencia forman estas relaciones débiles. Los puentes locales son conductores de energías entre grupos densa y fuertemente relacionados, pero aislados de su entorno, fracturados 21 .

Por el contrario, las relaciones débiles pluralizan y complejizan las opiniones, las actitudes de los actores sociales, haciéndolas menos dogmáticas y uniformes: «...los vínculos débiles, frecuentemente considerados como productores de alienación son vistos aquí como indispensables para las oportunidades individuales y para su integración en las comunidades; los vínculos fuertes, que reproducen la unión local, llevan a una fragmentación total. Las paradojas son un antídoto bienvenido para las teorías que explican todo cuidadosamente» (Granovetter, 1973;1.378).

En los análisis sobre el movimiento asociativo (y en el discurso más tradicional de las asociaciones), se suele mencionar el fraccionamiento producido en los últimos años, como si se tratase de una característica negativa. Es apropiado manifestar aquí la hipótesis, expuesta por J. Garrido (1996) de que, si las fracciones asociativas están vinculadas por puentes locales, harán fluir más energía entre toda la red, diversificará sus expresiones y cohesionará más a los diferentes grupos asociados. En vez de ser un argumento negativo puede resultar una bocanada de aire fresco para este movimiento social.

El sociograma, ya lo hemos mencionado en diferentes partes del texto, tiene sobre todo dos limitaciones fundamentales: es estático y descriptivo; describe estructuras estáticas, pero no procesos. Junto a estas limitaciones Jesús Ibañez menciona otra más: no aclara la relación entre la relación real y la representación de la relación:

«La relación real está doblemente representada: primero por una etiqueta verbal (en el test sociométrico la relación informal entre dos miembros es representada por la etiqueta «este me atrae» del test); luego esta etiqueta está representada por una figura (el grafo). Pero no se ponen a la luz las naturalezas de esas representaciones» (Ibáñez, 1990:20).

${ }^{21}$ No es extraño encontrarse, en las entrevistas sobre asociacionismo, alguna reticencia de la población no asociada a entrar en asociaciones, porque parece que van a invadir el espacio privado de un grupo. El núcleo de la asociación tiene fuertes relaciones que aparecen como un rechazo al interés de los neofitos por participar de ellas. 
Frente a estas limitaciones, innegables, he propuesto la realización de una secuencia de gráficos realizada de manera conjunta entre los sujetos de la investigación. La representación del proceso comienza a tomar forma, la relación entre la relación real y la representada está en proceso de construcción, a partir del proceso reflexivo en el que intervienen los actores de dichas relaciones. En la medida en que este proceso praxico tenga continuidad, las limitaciones se irán desbordando. Pero las premisas que establezco son: que los actores sean sujetos en la investigación, que el proceso práxico se refleje en los sociogramas (elaborados participadamente) y, como consecuencia, que se realice «transferencia de tecnología social» de los técnicos a los actores sociales.

En diversas investigaciones, nos hemos encontrado con que los diferentes ritmos de los actores hacían fluctuar su participación en aquéllas. La mirada compleja de este hecho nos ha de llevar a plantearnos que no hay sincronía absoluta, porque hay múltiples actores y múltiples sistemas de relaciones, de igual modo que hay múltiples relojes. El tiempo es una dimensión interna de los procesos históricos, no hay un tiempo absoluto (Prigogine) y sólo son sincrónicos aquéllos sucesos controlados por el mismo reloj. La construcción de relojes comunes es uno de los objetivos de los procesos participativos: llegar a encuentros en tiempos comunes.

Tampoco el ruido es una dimensión absoluta en los sistemas (Ibáñez, 1990:21); una información en un sistema es ruido en otro que no está en sincronía con aquél. Prestar más atención a los vínculos débiles, por los que fluye energía informacional, puede permitir a los sistemas que el ruido se vaya transformando en información y ésta en auto-eco-organización, en autonomía.

\section{BIBLIOGRAFIA}

Bautista, Antoni y Roviera, Bru (1998): «Nacionalistas vascos. La familia de los silencios», Magazine, diario La Vanguardia. Barcelona, 8 de Febrero. (pp. 38-47).

Barrow, John D. (1997): ¿Por qué el mundo es matemático?, Barcelona, Grijalbo Mondadori.

CONDE, Fernando (1994): «Procesos e instancias de reducción/formalización de la multidimensionalidad de lo real,...», en DELGADO y GUTIÉRREZ. Métodos y técnicas cualitativas de investigación social. Madrid, Técnos. (pp. 97-119).

Colectivo IOÉ (1987): «Los inmigrantes en España», en Documentación Social, n. 66. Madrid Cáritas Española. (pp. 320-323).

GarRIDO, Francisco J. (1996): Redes de acción social en Bogotá y Caracas. Tesis Doctoral. Universidad Complutense de Madrid (inédita).

GRANOVETTER, Mark (1973): «The strength of weak ties», en American Journal of Sociology; vol 78, n. 6 Mayo (pp. 1360-1380).

IBÁÑEz, Jesús (1986): Más allá de la sociología. Madrid, S XXI.

IBÁÑEZ, Jesús (1988): «Del pensamiento lineal al pensamiento complejo», en $A L F O Z, \mathrm{n}{ }^{\circ}$ 51 (pp. 13-18).

IвÁNEZZ, Jesús (1990): Nuevos avances en la investigación social (Investigación social de segundo orden). Suplementos Anthropos, 22.

IBÁÑEZ, Jesús (1994): Por una sociología de la vida cotidiana Madrid, Técnos. 
MANRIQue, Rafael (1990): «Estructuras disipativas. De la termodinámica a la terapia familiar», en Suplementos Anthropos, n. ${ }^{\circ} 22$, (pp. 75-77).

Martín GutiérRez, Pedro et alii (1995a): «Inmigrantes y rechazo: una IAP en la escuela», en Cuadernos de la Red, 3. Madrid, CIMS, (pp. 31-38).

Martín Gutífrez, Pedro (1995b): «Participación ciudadana: un estudio de casos referido a municipios», en Utopías, 164. Madrid, (pp. 71-78).

MARTín GutiérRez, Pedro (1998): "Mapas sociales y análisis de redes en la IAP», en Cuadernos de la Red, 5, (pp. 60-67).

MARTínez, Francisco J. (1988): "Complejidad», en Román ReYes (Dir.). Terminología Científico-Social. Barcelona, Anthropos, (pp. 131-136).

MAYNTZ, Renate, et alii. (1980): Introducción a los métodos de la sociología empírica. Alianza Universidad. Madrid. (2. ${ }^{\mathrm{a}}$ Edición) (pp. 159-174).

ORTí, Alfonso (1994): «La confrontación de modelos y niveles epistemológicos en la génesis de la investigación social», en Delgado y Gutiérrez, Métodos y técnicas cualitativas de investigación social. Madrid, Técnos, (pp. 85-95).

Otero Navascués, J. M. (1971): «Semblanza de Werner Heisemberg», en Futuro Presente, 2, (pp. 69-71).

Pearce, Barnett W. (1994): «Nuevos modelos y metáforas comunicacionales», en Fried Schnitman, Dora (Coord.) Nuevos paradigmas, cultura y subjetividad. Barcelona, Paidos, (pp. 265-283).

Pizarro, Narciso (1990): «Teoría de redes sociales», en Suplementos Anthropos, 22, (pp. 146-152).

Requena SAntos, Félix (1991): Redes sociales y mercado de trabajo. Monografías del CIS, N. ${ }^{\circ} 119$. CIS-Siglo XXI.

Requena Santos, Félix (1994): «Redes de amistad, felicidad y familia», en Revista Española de Investigaciones Sociológicas, 66, (pp. 73-90).

Requena Santos, Félix (1996): Redes sociales y cuestionarios. Cuadernos Metodológicos del CIS, 18.

Rodríguez GÓMEZ, Fernando (1997): Medio ambiente, desarrollo y paisaje en las sociedades post-industriales. Tesis Doctoral. Universidad Complutense de Madrid. (Inédita).

Rodríguez, Josep A. (1995): Análisis estructural y de redes. Cuadernos Metodológicos del CIS, N. ${ }^{\circ} 16$.

Sierra Bravo, R. (1988): Técnicas de investigación social. Paraninfo. Madrid. (5. ${ }^{a}$ Edición): (pp. 679-699).

STENT, Gunther (1990): «Las paradojas del universo», en Suplementos Anthropos, 22, (pp. 72-74)

VILlASANTE, Tomás R. (1991): Movimiento ciudadano e iniciativas populares. Madrid, Ed. HOAC.

VILlasante, Tomás R. (1993): «Aportaciones básicas de la I-A-P a la epistemología y la metodología», en Documentación Social, 92. Madrid, Cáritas Española, (pp. 23-41).

VILlASANTE, Tomás R. (1994 a): «De los movimientos sociales a las matodologías participativas», en Delgado y GutiérRez (Coord.) Métodos y técnicas cualitativas de investigación en ciencias sociales. Madrid, Editorial Síntesis. (pp. 399-424)

VILLASANTE, Tomás R. (Coord.) (1994 b): «Clientelas y emancipaciones, una introducción metodológica», en Las ciudades hablan. Caracas, Nueva Sociedad.

Villasante, Tomás R. (1995 a): «Metodología participantes y práxicas», en Cuadernos de la Red, 3. Madrid, CIMS, (pp. 4-10).

VILlasante, Tomás R. (1995 b): Las democracias participativas. Madrid, Ed. HOAC.

VV.AA. (1993): Curso sobre metodología de participación. CIMS-EDE-IOÉ (mecanografiado). 


\title{
RESUMEN
}

El sociograma, instrumento técnico que emana de la teoría de grafos y es aplicado en la teoría de redes, se muestra aquí como un elemento de trabajo en la intervención e investigación participante, siendo a su vez un cauce por el que los actores intervinientes visualizan su propia situación compleja. En el análisis de redes al uso el sociograma se elabora a partir de tests y matrices sociométricas; en este caso surge desde un plano no cuantitativo, mediante el análisis de textos y discursos, la intervención de los actores participantes en el proceso y con la intención de rearticular las redes sociales.

\begin{abstract}
The sociogramma, technical instrument that comes from the graph theory and is applied in network theory, is shown here as a tool in takingpart / participant investigation, being at the time a way for the actors in play to have a look at their own complex situation. In network analysis, the sociogramma is made from tests and sociometric matrices; in this case, it arises from a non cuantitative base, through texts and discourses analysis and the mediation of the actors participating in the process, with the purpose of rearticulate the social nets.
\end{abstract}

